\title{
Caracterização das teses e dissertações de enfermagem acerca do infarto do miocárdio
}

\author{
Raquel Pötter Garcia*, Maria de Lourdes Denardin Budó**, Mariane \\ da Silva Barbosa***, Bruna Sodré Simon*, Tifany Colomé Leal***, \\ Stefanie Griebeler Oliveira****
}

\begin{abstract}
Resumo: Objetivou-se delinear a tendência da produção científica acerca do Infarto do Miocárdio, com base nas teses e dissertações defendidas nos programas de pós-graduação em Enfermagem do Brasil. Revisão narrativa, com busca realizada no Banco de Teses e Dissertações da Coordenação de Aperfeiçoamento Pessoal de Nível Superior e nos Catálogos de Teses e Dissertações da Associação Brasileira de Enfermagem. A coleta de dados ocorreu no mês de janeiro de 2012, utilizando as palavras-chave "infarto agudo do miocárdio" e "infarto do miocárdio". Analisaram-se, quantitativamente, resumos de 27 produções cientificas. A análise qualitativa temática desenvolveu-se com 11 documentos, que foram agrupados nas seguintes categorias: Itinerário terapêutico dos pacientes; Fatores relacionados ao surgimento e manejo da doença; Infarto e saúde mental; Enfermagem e as atividades de educação em saúde. Verificou-se tendência quantitativa, demandando novas investigações que abarquem questões como os significados da doença, tornando possível desenvolver estratégias coerentes com as peculiaridades dos doentes pós-infarto.
\end{abstract}

Descritores: Enfermagem, Infarto do Miocárdio, Doença Crônica.

\section{Characterization of nursing dissertations and theses about acute myocardial infarction}

\begin{abstract}
This study aimed to delineate the trend of dissertations and theses about Acute Myocardial Infarction, based on theses and dissertations defended in the Nursing postgraduate programs in Brazil. It is a narrative revision, using a search in the Dissertations and Theses Database of the Higher Education Personal Development Coordination and in the Catalogues of Dissertations and Theses of the Brazilian Nursing Association. Data collection happened in January 2012, using the keywords "acute myocardial infarction" and "myocardial infarction". A quantitative analysis of 27 abstracts and a qualitative theme analysis of 11 documents were implemented, emerging the categories: Patients' therapeutic itinerary; Factors regarding the disease onset and management; infarction and mental health; Nursing and activities of health education. A quantitative trend was verified, demanding new investigations that approach themes such as meanings of the disease, enabling to develop strategies that consider peculiarities of the patients after the infarction.
\end{abstract}

Descriptors: Nursing, Myocardial Infarction, Chronic Disease.

*Mestranda do Programa de Pós-Graduação em Enfermagem na Universidade Federal de Santa Maria (UFSM), Santa Maria, RS, Brasil.

**Doutora em Enfermagem pela Universidade Federal de Santa Catarina (UFSC), Florianópolis, Brasil. Docente no Departamento de Enfermagem e no Programa de Pós-Graduação em Enfermagem da Universidade Federal de Santa Maria (UFSM), Santa Maria, RS, Brasil.

***Acadêmica de Enfermagem na Universidade Federal de Santa Maria (UFSM), Santa Maria, RS, Brasil.

****Doutoranda do Programa de Pós-Graduação em Enfermagem na Universidade Federal de Ciências Médicas de ISSN 2236-5843 Porto Alegre (UFCSPA), Porto Alegre, RS, Brasil. Docente na Universidade Federal de Pelotas (UFPel), Pelotas, RS, 107 Brasil. 


\section{Introdução}

A população brasileira, nos últimos anos, tem enfrentado uma transição demográfica devido à redução das taxas de fecundidade, de natalidade, e aumento da expectativa de vida. Esses fatos refletem no envelhecimento populacional e no surgimento de epidemias das doenças crônicas ${ }^{1}$, como as doenças cardiovasculares, neoplasias, doenças respiratórias crônicas e diabetes.

As doenças cardiovasculares são responsáveis por $31,3 \%$ do total de $72 \%$ dos óbitos que ocorrem pelas doenças crônicas, representando a maior causa de morte no país. ${ }^{1-2} 0$ Infarto do Miocárdio (IM) é uma síndrome clínica resultante da necrose isquêmica do músculo cardíaco, consequente da obstrução do fluxo coronariano, transitória ou permanentemente. A maioria dos IMs são ocasionados pela trombose de uma artéria coronária sobre uma placa ateromatosa. ${ }^{3}$ Essa placa é formada ao longo do tempo e pode romper, causando hemorragia, fissura ou ulceração, o que obstrui parcial ou totalmente 0 suporte de oxigênio e suprimentos ao coração, desencadeando a isquemia miocárdica. ${ }^{4}$

Desse modo, a doença coronária aguda não se caracteriza por fatores isolados, demandando o envolvimento de vários setores da sociedade para atenuar seus efeitos. Dentre esses, a prática baseada em evidências pode proporcionar sucesso na melhoria dos cuidados e maior adequação ao tratamento. ${ }^{5}$ Estudos nacionais e internacionais que façam um levantamento das especificidades das pesquisas já desenvolvidas relativas à temática podem ser benéficos no sentido de comparar achados, bem como detectar possiveis alterações e propostas que podem ser (re) adequadas a prática clínica. ${ }^{6}$

Diante dessas considerações, se faz necessário, que a Enfermagem atente para as questões que permeiam o IM, uma vez que essa doença ainda se apresenta com altos índices de incidência e prevalência na população, o que demanda o conhecimento de suas peculiaridades para uma assistência com melhor qualidade. Ainda, ao difundir a produção existente das dissertações e teses sobre a temática e destacar suas características, pode-se proporcionar subsídios para a elaboração de novos trabalhos que sejam capazes de sustentar a prática de enfermagem com essa população, bem como oferecer suporte para que os enfermeiros e seus pares atuem nos serviços de saúde pautados nas evidências científicas já encontradas.

A relevância desse estudo concentra-se na necessidade de aprofundar o que se tem produzido na área, facilitando, desse modo, a disseminação de informações em âmbito acadêmico e profissional, por meio do artigo científico, o qual possui, normalmente, maior facilidade de acesso do que as teses e dissertações. Desse modo, a questão norteadora desta pesquisa foi: Qual a tendência das teses e dissertações acerca do IM na Enfermagem? E objetivou-se delinear a tendência das teses e dissertações sobre IM defendidas nos programas de pós-graduação em Enfermagem (PPGEnf) do Brasil.

\section{Metodologia}

Saúde (Santa Maria), v.38, n.2, p. 107-122, 2012. Caracterização das teses e dissertaçöes de enfermagem acerca do infarto do miocárdio
Estudo de revisão narrativa, descritivo. Esse tipo de revisão é utilizado para descrever e discutir determinadas temáticas, pautando-se nas publicações impressas ou eletrônicas, diante da interpretação e análise crítica dos autores. ${ }^{7}$ 
A busca das publicações foi realizada no Banco de Teses e Dissertações da Coordenação de Aperfeiçoamento Pessoal de Nível Superior (CAPES) e também nos Catálogos de Teses e Dissertações da Associação Brasileira de Enfermagem (ABEn). Optouse pela procura de somente esses documentos, devido à necessidade de visibilizar a produção existente acerca da temática nos programas de pós-graduação e evitar 0 desenvolvimento de uma nova pesquisa semelhante. Além disso, algumas dissertações e teses recentes, ainda não possuem artigos científicos publicados, o que impede a divulgação do conhecimento. Salienta-se que essa busca foi oportunizada por uma disciplina, de um PPGEnf, de uma instituição de ensino do interior do Rio Grande do Sul.

A coleta de dados ocorreu em janeiro de 2012. No formulário de pesquisa da CAPES utilizaram-se, separadamente, as palavras-chave "infarto agudo do miocárdio" e "infarto do miocárdio". Na ABEn, mesmo não havendo formulários para busca, foram procurados os mesmos termos da CAPES, nos títulos e resumos das teses e dissertações disponíveis nos catálogos.

Realizou-se a busca sem delimitar um recorte temporal, a fim de alcançar um maior número de produções existentes sobre a temática. No entanto, os catálogos da $A B E n$ encontram-se disponíveis somente no período de 2001 até 2010. Dessa forma, para selecionar, inicialmente, as produções, estabeleceram-se os seguintes critérios de inclusão: ser da temática do IM; ser pesquisa desenvolvida por enfermeiros ou por PPGEnf e ter resumo disponível online, na íntegra e gratuitamente. Como critérios de exclusão: tese ou dissertação sem resumo ou com incompletude.

A seleção ocorreu por meio da leitura dos títulos e resumos das teses e dissertações. Foram encontrados, na CAPES, 481 documentos com a palavra-chave "infarto agudo do miocárdio", dos quais foram selecionados 20. Com a palavra-chave "infarto do miocárdio", foram encontradas 751 produções, sendo selecionadas 39, porém apenas três não estavam repetidas na busca com a palavra-chave "infarto agudo do miocárdio". Assim, o total de documentos selecionados, na CAPES, foi de 23 (20 encontrados com a primeira palavrachave, e três, com a segunda). Na ABEn foram localizadas 12 produções, mas selecionaramse apenas quatro, pois as outras oito já constavam na CAPES.

Por fim, de acordo com a adequação aos critérios de inclusão, analisaram-se quantitativamente os resumos de 27 produções científicas, entre teses e dissertações. Para a descrição dessas produções, elaborou-se um quadro sinóptico, constituído das variáveis: tipo (tese ou dissertação); ano de publicação; instituição de origem; região; cenário e sujeitos do estudo; e abordagem metodológica (qualitativa ou quantitativa). A caracterização das teses e dissertações foi realizada por meio da utilização do cálculo da frequência relativa.

A classificação da abordagem metodológica ocorreu mediante termos explícitos no método dos estudos, sendo que aqueles que não destacavam claramente 0 tipo de abordagem buscaram-se detalhes metodológicos que poderiam se referir aos estudos quantitativos ou qualitativos, como por exemplo, pesquisas identificadas do tipo coorte, casocontrole e clínico-randomizado. Ainda, para determinar os sujeitos pesquisados foi feito um levantamento em cada estudo da população investigada.

A análise qualitativa foi realizada somente com as teses e dissertações encontradas na íntegra online e gratuitamente, totalizando 11 documentos. Estes foram localizados no portal da universidade em que a pesquisa foi realizada, por meio do buscador Google e também por contato eletrônico com os autores. De modo a auxiliar na análise, utilizou-se uma ficha de

Saúde (Santa Maria), v.38, n.2, p. 107-122, 2012. Garcia, R. P., et al. ISSN $2236-5843$ 109 
extração de dados das variáveis, contendo os resultados e conclusões/recomendações relacionados ao objeto do estudo. A análise desses dados ocorreu por meio da análise temática ${ }^{8}$, a qual se constitui de três etapas: pré-análise, exploração do material, e tratamento dos resultados obtidos e interpretação.

A análise qualitativa permitiu a inferência do surgimento de algumas temáticas comuns nas teses e dissertações, possibilitando a elaboração das categorias: O itinerário terapêutico dos pacientes; Fatores relacionados ao surgimento e manejo da doença; Infarto e Saúde mental; Enfermagem e as atividades de educação em saúde.

\section{Resultados}

Inicialmente, serão apresentadas as características gerais dos 279-35 estudos por meio de descrição; as quais possibilitam vislumbrar a tendência que esses apresentam. Em seguida, serão discutidos os principais dados provenientes dos resultados e conclusões/recomendações relacionados às $11^{9-19}$ teses e dissertações encontradas na integra online e gratuitamente.

Dos 27 documentos selecionados, 77,78\% corresponderam a dissertações, e 22,22\%, a teses. Estas se apresentaram no período entre os anos de 1991 e 2010, sendo que 0 ano de maior número de produções foi do ano de 2000 , com $14,81 \%$ teses e dissertações.

Os trabalhos originaram-se de diversas instituições de ensino do país. 0 dado mais representativo foi proveniente da Escola de Enfermagem da Universidade de São Paulo (USP) - Ribeirão Preto, com 29,62\%. Todas as outras instituições ficaram com o número de publicações abaixo de 15\%. Dessa forma, a Região que prevaleceu nas publicações dessa temática foi a Sudeste, com $55,55 \%$, seguida das Regiões Sul e Nordeste, com $22,22 \%$ cada uma.

O cenário de destaque, nos estudos, foi o hospitalar9-11, 14, 16-23, 27-31, 33, com 66,67\% das publicações, incluindo-se nele unidades coronarianas ou cardiológicas, enfermarias cardiológicas, unidades de terapia intensiva e centros coronarianos. Ainda ressaltam-se os ambulatórios de cardiologia ${ }^{12-13,26,32,34-35}$, os quais, mesmo que característicos do ambiente hospitalar, foram especificados em $22,22 \%$ dos estudos; houve também $3,7 \%$ dos estudos realizados em clínica cardiológica particular. ${ }^{15} \mathrm{Em} 7,4 \%$ dos estudos, 0 resumo não contemplava informação sobre o cenário do estudo. ${ }^{24-25}$

Quanto ao tipo de abordagem metodológica, 22,22\% das publicações caracterizaram-se como estudos qualitativos ${ }^{15,}$ 20, 22, 26-28, e 66,67\%, como quantitativos. ${ }^{9-14}, 16-19,21,23-24,29-30,32-34$ Alguns, correspondentes a 7,4\%, tratavam de implementação de referencial teórico e elaboração de marco conceitual ${ }^{31,35}$, fator definido pela leitura da própria metodologia dos estudos. Destaca-se que apenas 3,7\% dos estudos apresentou abordagem mista ${ }^{25}$, ou seja, quanti/qualitativa.

Quanto aos sujeitos pesquisados, 62,96\% eram pacientes que tiveram IM e estavam internados $9-11,14-17,19-23,28-31,33 ; 29,62 \%$ também haviam tido IM, porém não estavam mais

Saúde (Santa Maria), v.38, n.2, p. 107-122, 2012. Caracterização das teses e dissertaçōes de ISSN 2236-5834 internados $^{12-13,25-27,32,34-35}$ e, ainda, 3,7\% corresponderam à família ${ }^{24}$ do paciente pós IM; outro estudo abordou as características desse tipo de paciente, no prontuário. ${ }^{18}$

Para tanto se demonstra uma tendência de estudos provenientes de programas de mestrado e elevado índice de produção a partir do ano de 2000. A região Sudeste é destaque 
com o maior numero de estudos realizados. 0 cenário hospitalar, como local, e os pacientes pós IM internados, como sujeitos de pesquisa, são a tendência dos estudos; assim como, a abordagem quantitativa.

\section{Itinerário terapêutico dos pacientes}

Em alguns estudos evidenciou-se que ocorre uma demora na busca de assistência, sendo que o principal motivo para esse atraso está relacionado ao julgamento incorreto dos sintomas ${ }^{9-10}$, pois os pacientes realizam automedicação ou acreditam que logo vão melhorar. ${ }^{10}$ No momento do infarto, alguns pacientes estavam sozinhos, porém, a maioria encontrava-se na companhia do(a) companheiro(a) ou dos filhos. Quanto às ações imediatas, destacam-se a procura por atendimento médico, o estímulo à ingesta de líquidos e 0 descanso para alívio dos sintomas. ${ }^{10}$

Os locais procurados para atendimento, em alguns casos, demonstram-se como inadequados, favorecendo a demora para resolução do problema. ${ }^{9}$ Dentre aqueles que acompanharam os doentes até o serviço de saúde estavam os vizinhos, os filhos, companheiros ou outros familiares. ${ }^{10}$

Estudos trazem que alguns pacientes que tiveram 0 infarto já haviam, em algum momento, procurado auxílio médico, 11-13, estando em acompanhamento, principalmente, para o controle da diabetes, hipertensão ou outro problema cardíaco. ${ }^{12,}{ }^{14-15}$ Além disso, alguns já tinham sofrido infartos prévios. ${ }^{10,13,16-18}$

\section{Fatores relacionados ao surgimento e manejo da doença}

Quanto à idade, grande parte dos estudos caracteriza os doentes com intervalo entre 50 e 60 anos. ${ }^{10-12,14,17-19}$ Ao se referir ao sexo e escolaridade, as pesquisas são unânimes, pois a maioria dos indivíduos que infartaram eram homens e estudaram até 0 ensino fundamental. Outra questão coincidente em todos os estudos foi o estado civil, sendo, na maior parte, casados.

A raça e religião não foram abordadas em todas as pesquisas, porém, quando destacadas, a raça que mais apareceu foi a branca ${ }^{12,15,18}$, seguida da negra-10 e parda' ${ }^{13,19}$; a religião foi a católica. ${ }^{12,19}$ Ao se referir às comorbidades, somente uma dissertação não falou sobre elas ${ }^{19}$; a Hipertensão Arterial Sistêmica (HAS) e a Diabetes Melittus (DM) estiveram presentes em todos os outros estudos. Além disso, ressaltam-se o tabagismo, dislipidemia, histórico familiar, sedentarismo, obesidade, etilismo, estresse e acidente vascular cerebral prévio.

No que se refere ao conhecimento da doença, os indivíduos apresentam dificuldades quanto à compreensão de sintomas que antecedem o período do infarto ${ }^{10,15}$ e do processo de doença. ${ }^{14}$ Apesar disso, alguns fatores de risco, como a HAS e DM, são conhecidos como possíveis causadores do $\mathrm{IM}^{12,14}$, o que faz com que algumas pessoas utilizem terapias alternativas, como remédios caseiros ${ }^{12}$, para auxiliar no manejo dessas doenças e também prevenir possíveis complicações.

Saúde (Santa Maria), v.38, n.2, p. 107-122, 2012 Garcia, R. P., et al.

Ainda, em alguns casos, os sujeitos relataram que conheciam os fatores de risco e agravantes da doença, mas nem por isso deixavam de se expor a eles. ${ }^{14}$ Dessa forma, logo após o infarto e a internação hospitalar, os indivíduos pensam em modificar seus hábitos de 
vida, mas ressaltam que esse fato é difícil. ${ }^{13,} 18$ Há dificuldade para compreender a cronicidade da doença, o que faz com que, no início, existam mais práticas para melhoria das condições de saúde, porém, com a melhora dos sintomas, volta-se à rotina normal. ${ }^{15}$

\section{Infarto e saúde mental}

Quanto aos aspectos relativos à saúde mental, os estudos abordam que os pacientes acreditam que o IM ocorreu devido ao estresse que estavam vivenciando na sua rotina diária de vida. ${ }^{12-16,18}$ Dentre os tipos de estresse que podem ter causado o IM, há relatos que se referem ao trabalho ${ }^{12-15}$, preocupações com os filhos, problemas financeiros, familiares ${ }^{12} \mathrm{e}$ emocionais..$^{15}$ Outros abordam ainda, a ansiedade como fator predisponente ao infarto. ${ }^{12-13 \text {, }}$ 15,18

Além das questões vinculadas à saúde mental que podem ter causado o IM, há também aquelas referentes ao pós $\mathrm{IM}$, as quais geram sentimentos de medo, angústia e insegurança quanto ao ocorrido e as suas consequências na vida. ${ }^{12,15,19}$ Essas podem se relacionar a internação, procedimentos hospitalares, afastamento da família, do trabalho, bem como as imposições de mudanças de hábitos e o medo para prognóstico ruim da doença, inclusive a morte. ${ }^{10,16,18-19}$

Um ponto de destaque nos estudos foi à depressão, sendo integrante tanto do pré, quanto do pós-infarto. No período anterior, essa é considerada como um dos fatores de risco que podem auxiliar no desencadeamento do infarto, sendo associada com conflitos que as pessoas vivenciam na rotina. ${ }^{12}$ Por outro lado, posteriormente, a depressão pode aparecer devido a não aceitação da cronicidade da doença, das limitações causadas pela mesma e do desejo de morte. ${ }^{12,18}$

Dessa forma, há necessidade do reconhecimento desse possivel problema no pósinfarto, pois se controlado pode favorecer na melhoria do estado de saúde ${ }^{11}$, diminuir a mortalidade e aumentar a sobrevida do paciente. ${ }^{18}$ Ainda, a família foi destacada por alguns estudos como forma de apoio nessa etapa, a qual deve ser inserida nas orientações da enfermagem, a fim de que possa auxiliar na melhor adaptação frente à nova condição de vida, reduzindo sintomas de ansiedade e depressão ${ }^{14}, 18$, bem como incentivando no acompanhamento do tratamento. ${ }^{12}$

\section{Enfermagem e as atividades de educação em saúde}

A educação em saúde aparece como prevenção, sobretudo, para doenças como HAS, DM, dentre outras. ${ }^{14}$ Para isso, os serviços de saúde devem elaborar programas educativos específicos, a fim de melhorar a assistência, promover a saúde e evitar agravos cardiovasculares. $., 14,19$ Destaca-se que grande parte dos indivíduos acometidos por uma doença cardiovascular desconhece as maneiras corretas de lidar com a doença, sobretudo, porque recebem precárias orientações dos profissionais de saúde. ${ }^{12-14}$

Quanto ao período de internação, as atividades de educação em saúde objetivam tornar

Saúde (Santa Maria), v.38, n.2, p. 107-122, 2012. Caracterização das teses e dissertaçöes de enfermagem acerca do infarto do miocárdio o paciente coparticipante no gerenciamento de sua doença e assim favorecer sua reabilitação. ${ }^{14}$ Essas estratégias visam o desenvolvimento da autonomia, no intuito de que os pacientes possam conhecer seus problemas e, a partir de sua realidade, modificar hábitos para atender às imposições da doença cardiovascular. ${ }^{18}$ 
A educação em saúde promove 0 fornecimento de informações aos pacientes ${ }^{17}$, cabendo à enfermagem atentar para as modificações no seu estilo de vida, a fim de auxiliar conjuntamente nesse processo de adaptação. ${ }^{15}$ As ações devem ser direcionadas e implementadas por meio do processo de enfermagem, o que facilita um cuidado voltado às necessidades do paciente, atendendo-o de forma integral e individualizada. ${ }^{19} \mathrm{O}$ conhecimento dos fatores subjetivos da clientela pode auxiliar até mesmo na melhora de resposta dos serviços de emergência, visando à aplicação de cuidados imediatos quando um evento cardiovascular está em curso ${ }^{9}$.

\section{Análise e discussão dos dados}

O primeiro programa de pós-graduação stricto sensu, com mestrado acadêmico em Enfermagem, do Brasil, surgiu em 1972, na Escola de Enfermagem Anna Nery, seguido pela USP - Ribeirão Preto, no ano de 1973. Já o primeiro curso de doutorado foi inaugurado somente em 1981, na USP - Ribeirão Preto. ${ }^{36}$ Os dados encontrados podem sugerir 0 pioneirismo dessas universidades, bem como o privilégio de alguns estados brasileiros, localizados na Região Sudeste ${ }^{37}$, pois são potenciais fontes de pesquisadores e publicações, o que pode justificar o número maior de publicações nesses locais. Porém, também destacase que alguns programas de pós-graduação podem trabalhar com outras temáticas e por isso não apareceram nas buscas realizadas nessa revisão.

Apesar disso, observa-se uma tentativa de expansão do aparato científico e tecnológico para outras regiões do país, a fim de descentralizar e viabilizar o progresso para toda a população brasileira ${ }^{37}$, de forma igualitária. Foi na década de 90 que a pós-graduação se consolidou e, posteriormente, no ano 2000, houve a criação do Mestrado Interinstitucional pela CAPES, favorecendo o convênio com escolas que ainda não tinham programas ${ }^{38}$, contribuir de certa forma, para a expansão das pesquisas em enfermagem a partir dessa época.

Sobre a temática do IM, o número elevado de produções, nesse período, pode ter ocorrido uma vez que na década de 90 os dados epidemiológicos referentes às doenças cardiovasculares, especificamente o IM, ainda eram elevados. Assim, no início dos anos 2000 novos métodos diagnósticos e farmacológicos ${ }^{39}$ foram implementados, o que talvez suscitou a realização de mais estudos.

O hospital se caracterizou como cenário representativo nas investigações. A busca maior pela realização das pesquisas neste ambiente pode ter advindo à ideia de que o infarto é uma doença aguda. Assim, posteriormente, o doente vivenciaria ou significaria a enfermidade de maneira diferente de quando ocorreu o processo de agudicidade, o que impediria de se verificar a real percepção dos envolvidos, fazendo com que o interesse dos pesquisadores se volte mais para o período de internação. Um estudo, realizado com pacientes pós IM, ainda internados detectou que, embora os pacientes tivessem um grande envolvimento em seu tratamento e recuperação quando hospitalizados, no período posterior os cuidados passavam a reduzir, demonstrando que a internação é diferente do momento posterior, quando o indivíduo já se encontra recuperado e com mais possibilidades de agir conforme o que acha coerente ${ }^{40}$.

Saúde (Santa Maria), v.38, n.2, p. 107-122, 2012 Garcia, R. P., et al. 
No entanto, mesmo que alguns eventos cardiovasculares sejam agudos, os seus cuidados permanecem ao longo da vida ${ }^{5}$, desencadeando, muitas vezes, no paciente e família grande sofrimento emocional. ${ }^{41}$ Tal fato se confirma também pela realização de algumas das pesquisas ${ }^{12-13,26,32,34-35}$ encontradas neste estudo, nas quais os pacientes ficam em acompanhamento nos ambulatórios de cardiologia devido ao acometimento cardiovascular.

Outro dado relevante foi o número significativo de pesquisas quantitativas. Para esse dado podem-se relacionar duas questões: uma delas é que a abordagem quantitativa teve seu surgimento anterior à qualitativa, sobretudo, por influência do positivismo. Nas décadas de 40 a 60 do século XX, os textos traziam valores e critérios positivistas que deveriam ser testados por meio da estatística. A pesquisa qualitativa, nesse período, considerava-se algo a ser realizado após a pesquisa quantitativa. ${ }^{42}$

A outra questão se refere ao fato de que o IM é uma enfermidade com histórico de estudos na área da medicina, uma vez que é uma doença com alta morbimortalidade e por isso tem sido estudada para descoberta de tecnologias que favoreçam a melhora do prognóstico e a sobrevida dos pacientes. Diante disso, a medicina se caracteriza por desenvolver basicamente estudos quantitativos, com influência das ciências naturais ${ }^{43}$, o que talvez estimulasse na realização do grande número de pesquisas quantitativas pela enfermagem, nessa temática, visando à descoberta de fatores de risco e comportamentais.

Os sujeitos das publicações foram em sua maioria os pacientes pós IM, o que caracteriza apenas um viés que envolve a doença. A família foi descrita em somente um estudo, fato que evidencia a necessidade de novas investigações na área, pois esta vivencia, juntamente com o doente, a adaptação e a trajetória a ser percorrida pelas imposições do processo saúde/doença. No adoecimento crônico os membros da família são afetados, a organização e o funcionamento da dinâmica familiar também se alteram para a busca do equilíbrio. ${ }^{44}$ Desse modo, sugerem-se estudos que apresentam a visão dos enfermeiros e do restante da equipe de saúde frente à assistência aos pacientes pós IM, já que estes vivenciam também a rotina à qual os doentes são submetidos após esse acometimento.

Com relação a categoria do itinerário terapêuticos foi evidenciado que as pessoas pouco acreditam que uma doença como o infarto irá ocorrer e, desse modo, subjugam os sinais e sintomas do adoecimento, mesmo que, às vezes, estes se iniciem precocemente. Nesse contexto, acredita-se em duas possibilidades. Uma é que, se os doentes estão sozinhos, podem tentar amenizar a situação pelo uso de alternativas ${ }^{10,46}$ que auxiliem na redução dos sintomas, até que, no momento da agudicidade, não conseguem buscar auxílio, tornando, muitas vezes, o episódio fatal. Assim, a busca pelo atendimento ocorre de maneira tardia, seja para atenuar os fatores de risco, como a HAS, ou para resolver a crise no momento em que o IM está acontecendo.

Porém, se os indivíduos estão acompanhados por algum conhecido, podem buscar ajuda mais rapidamente, pois, geralmente, o outro fica preocupado em ver seu ente querido enfermo. Por outro lado, mesmo que os sinais e sintomas do IM já estejam incorporados no saber popular, existe a possibilidade de os indivíduos e seus familiares agirem conforme suas crenças para tentar resolver os problemas de saúde no ambiente domiciliar.

Desse modo, em um primeiro momento, não se deslocam para o setor profissional, e

Caracterização das teses e dissertaçōes de enfermagem acerca do infarto do miocárdio realizam práticas que acreditam que possam ser resolutivas, como o uso de chás e automedicação. $\mathrm{O}$ ambiente domiciliar proporciona condições próprias de agir, as quais são construídas no decorrer da vida. Assim, há uma tentativa de tratamento, mesmo que este esteja relacionado ao saber profissional, como o uso da medicação. ${ }^{45-46}$ 
Quando as atitudes desenvolvidas no setor popular não são efetivas, os sujeitos passam a ficar preocupados e, com isso, deslocam-se para os serviços de atendimento. 0 itinerário pode se iniciar por qualquer ponto da rede de assistência e, dependendo do local, as probabilidades de resolutividade para a doença podem se reduzir. Mesmo que as crises de dor causem sofrimento prolongado, buscam-se soluções no setor popular e farmacêutico antes do profissional. ${ }^{46}$

Outra questão a destacar, e que exige atenção dos profissionais de saúde, é que, de alguma forma, os doentes já estão inseridos nos serviços de saúde, seja por tratamento dos fatores de risco ou por infartos anteriores. O IM se caracteriza como um "evento sentinela", o qual surge devido às falhas nos serviços de saúde, e que poderia ser evitado se as ações, sobretudo, na atenção básica, considerassem as representações dos indivíduos que experienciam a doença, suas crenças e significados, as quais estão impregnadas no processo de vida. ${ }^{46}$ Desse modo, há necessidade de focar as ações de saúde nas peculiaridades de cada usuário, pois as pessoas, normalmente, agem de acordo com o que consideram importante para sua vida e assim podem deixar até de utilizar a medicação, uma vez que por algum motivo isso não faça sentido.

As pessoas fazem escolhas, conforme o universo sociocultural a que pertencem; são mediadas pelo atual problema de saúde, as experiências anteriores, bem como os planos futuros de vida. ${ }^{46}$ Assim, mesmo que os profissionais de saúde estabeleçam práticas necessárias para a conduta dos pacientes, estes desenvolvem sua maneira de cuidado, sendo influenciados pela inter-relação entre o saber popular e científico. ${ }^{44}$ Para tanto, almeja-se que ocorra um movimento desses profissionais para a adoção de práticas que façam sentido para a população, tornando o enfrentamento do processo saúde/doença relevante para essas pessoas.

Na segunda categoria, que trata dos fatores relacionados ao surgimento e manejo da doença pode ser detectado que todos os estudos analisados qualitativamente abordaram de alguma forma questões relativas ao perfil sociodemográfico e de saúde dos indivíduos que tiveram IM. Dentre as principais questões destacadas no perfil desses indivíduos estão idade, sexo, escolaridade, renda familiar, raça, estado civil, religião e comorbidades. Desse modo, conhecer esses fatores, auxilia no planejamento das ações de intervenção dos profissionais de saúde, o que facilita para um atendimento mais eficaz e específico.

Os fatores que podem auxiliar no desencadeamento de uma doença cardiovascular são os mais variados e têm sido estudados continuamente, uma vez que, de acordo com 0 avanço da tecnologia, novas possibilidades podem ser descobertas. Nesse sentido, a maioria dos estudos analisados considera imperativo abordar os fatores de risco, já que, conforme a época e o contexto em que são realizados podem ser diferentes do que já se tinha estabelecido por pesquisas anteriores.

Apesar disso, atualmente, sabe-se, de maneira assertiva, que o acometimento cardiovascular é multicausal ${ }^{47}$ e influenciado por questões desenvolvidas ao longo da vida. Como toda doença crônica, o IM possui um extenso período anterior até desencadear 0 episódio principal, sendo os fatores de risco coadjuvantes no processo.

Os condicionantes que irão determinar 0 aparecimento da aterosclerose $\mathrm{e}$ consequentemente a cardiopatia isquêmica são multifatoriais e devem ser identificadas a fim de subsidiar as intervenções necessárias. ${ }^{47}$ Existem os fatores de risco modificáveis ${ }^{2}$, relacionados com o estilo de vida e os não modificáveis, que os indivíduos carregam consigo

Sáde (Santa Maria), v.38, n.2, p. 107-122, 2012 Garcia, R. P., et al. ISSN 2236-5843 115 
no decorrer da vida. Dentre os não modificáveis está o sexo, uma vez que, de acordo com os estudos analisados, neste artigo, foi possível identificar que a maioria dos sujeitos que infartaram eram homens.

Esse fato tem se modificado, pois homens e mulheres desenvolvem de forma semelhante o IM, porém a doença afeta o sexo feminino em idade mais avançada. Alguns fatores indicam maior risco para as doenças cardíacas nas mulheres, como o uso do tabaco e altos níveis de triglicerídeos, sendo esta uma diferença entre os sexos. Ainda, a diabetes, a obesidade e a depressão são mais prevalentes na população feminina. ${ }^{48}$

O estresse, fato revelado nos dados da terceira categoria que fala do IM e saúde mental, tem se caracterizado como um problema atual, pois as pessoas vivenciam cotidianamente ocasiões geradoras de conflitos. Essas situações intensificam a ansiedade, que se não controlada, em longo prazo poderá interferir seriamente na qualidade de vida dos sujeitos, favorecendo um envelhecimento problemático. Por outro lado, o estresse psicológico é difícil de avaliar, pois ainda está em discussão se um sofrimento dessa natureza contribui significativamente para o desenvolvimento da aterosclerose ${ }^{49} \mathrm{e}$ consequentemente $\mathrm{o} I \mathrm{IM}$.

Ainda, a própria internação hospitalar pode gerar ansiedade e desequilíbrio para 0 paciente e sua família, ainda mais quando a doença aparece de forma repentina como o IM. Não há um preparo para esse momento e por isso ocorre a necessidade de se refazer 0 equilíbrio. Os membros da família são afetados e a organização e o funcionamento da dinâmica familiar se alteram também para a busca de estabilidade novamente. ${ }^{44}$ Ressalta-se que a mudança pode ser benéfica ou não, sendo nesse caso considerada, de certa forma positiva, pois poderá estimular o doente e sua família na aquisição de hábitos mais saudáveis que auxiliem na melhora do bem-estar de todos.

No entanto, algumas modificações como o afastamento das atividades laborais, alterações nos hábitos alimentares e adesão ao tratamento medicamentoso, em um primeiro momento, inquietam 0 indivíduo. Essas podem dificultar o equilibrio, causar eventos estressores, solidão e até mesmo depressão. Aponta-se que os níveis mais elevados de estresse emocional observados em idosos estão associados com a doença cardíaca, ou seja, não acontecem em função do envelhecimento. Pacientes pós IM apresentaram alto nível de ansiedade e depressão em relação à pesquisa realizada com um grupo de idosos saudáveis. ${ }^{50}$

Nesse caso, destaca-se que a rede social de apoio, seja a família, os amigos ou colegas de profissão, dentre outros, têm papel importante, pois contribui para a minimização dos efeitos da doença na vida dos sujeitos pós IM. $\mathrm{O}$ apoio social configura-se como um produto dos relacionamentos sociais que as pessoas estabelecem entre si. Esse é acionado em momentos difíceis, como no surgimento de uma condição crônica. ${ }^{51}$

Por meio da última categoria, a Enfermagem e as atividades de educação em saúde visualiza-se que a maioria dos indivíduos acometidos por uma doença cardiovascular inesperada, como o IM, não estão preparados para enfrentar as dificuldades dessa nova condição de vida. Assim, torna-se fundamental o papel do enfermeiro como educador e facilitador do processo saúde/doença. Diante disso, ressalta-se que as práticas educativas devem fazer parte do trabalho diário dos enfermeiros, com o intuito de não somente prevenir doenças, mas também promover saúde e auxiliar na melhora da qualidade de vida dos 
Impera-se que o enfermeiro facilite a compreensão das pessoas quanto a sua condição de cronicidade, proporcionando informações sobre o tratamento, prevenção e promoção da saúde, bem como alternativas de acesso disponíveis na rede de assistência em saúde. Essa rede de apoio deve conter profissionais treinados e qualificados, acesso a serviços de saúde, bem como referência e con-trarreferência. ${ }^{52}$ É nesse contexto que 0 enfermeiro pode atuar, por meio do compartilhamento de saberes com o paciente e sua família, desde o momento de submissão, na emergência, passando pela internação hospitalar e chegando ao período posterior ao infarto.

Nesse sentido, uma comunicação eficiente entre paciente/família e equipe de saúde torna-se relevante para que possam ser reconhecidas as potencialidades de cada sujeito e a partir disso se pensar na elaboração de ações que atendam suas necessidades. A Enfermagem, por desenvolver cuidados próximos aos doentes, necessita encontrar elementos que a auxiliem na abordagem familiar, pois os aspectos do cotidiano interferem no processo saúde/doença. ${ }^{45}$

A realização de práticas sensíveis promovem a comunicação, 0 que facilita 0 estabelecimento de vínculos de confiança que serão responsáveis pela realização de atividades coerentes com a realidade dos indivíduos. ${ }^{45} \mathrm{O}$ diálogo e a escuta qualificada se destacam nesse processo, se configurando como ferramentas que favorecem a aproximação entre os sujeitos envolvidos.

\section{Conclusão}

Compreende-se que o IM proporciona uma série de modificações, tanto na vida do indivíduo com a doença, como de sua família. Essas alterações ocorrem diante da necessidade de readaptação para vivenciar essa nova maneira de vida. Assim, as pesquisas com abordagem qualitativa podem ser mais eficazes para a visualização dos significados que esse novo processo de viver proporciona aos indivíduos.

Porém, ao realizar este trabalho, observou-se que há um reduzido índice de dissertações e teses, com abordagem qualitativa, referentes à temática, caracterizando-se como lacuna da construção do conhecimento. Desse modo, expõe-se a necessidade de ampliar o número de publicações com essa abordagem, para que, a partir da identificação dos significados, possam ser elaboradas novas formas para o controle de infarto e reinfarto. Ainda, essas pesquisas poderiam auxiliar em uma educação em saúde pautada no compartilhamento de informações entre profissionais de saúde e usuários/família.

No entanto, foi possível comprovar a tendência nos estudos de que as produções da pós-graduação em enfermagem sobre o IM estão voltadas ao itinerário terapêutico percorrido pelas pessoas afetadas por esse adoecimento; aos fatores que ocasionaram e o manejo do IM; a relação do IM com a saúde mental, e por fim, as ações da enfermagem mediante as atividades de educação em saúde com os sujeitos e familiares que convivem com essa condição crônica.

Destaca-se, como fator limitante deste estudo, a indisponibilidade de algumas dissertações e teses no formato eletrônico, sobretudo, as anteriores ao ano de 2000, o que por um lado pode ter fragilizado os achados, fazendo com que a tendência do estudo não tenha em sua descrição todas as facetas possíveis. Porém, os dados encontrados sugerem

Saúde (Santa Maria), v.38, n.2, p. 107-122, 2012 Garcia, R. P., et al. 
que outras regiões e estados brasileiros busquem o desenvolvimento de novos estudos sobre $\mathrm{IM}$, pois assim poderão ser detectadas as necessidades específicas da população de cada localidade, a fim de proporcionar subsídios coerentes para atuação dos enfermeiros, bem como favorecer 0 estabelecimento de práticas voltadas à melhora da qualidade de vida dos sujeitos pós-infarto do miocárdio e suas famílias.

\section{Referências bibliográficas}

1. Brasil. Ministério da Saúde. A vigilância, o controle e a prevenção das doenças crônicas não transmissiveis: DCNT no contexto do Sistema Único de Saúde Brasileiro. Organização Pan-Americana da Saúde, Brasilia, 2005, $1^{\text {a }}$ edição. 80 p.

2. Brasil. Ministério da Saúde. Secretaria de Vigilância em saúde. Departamento de Análise de Situação de Saúde. Plano de ações estratégicas para o enfrentamento das doenças crônicas não transmissiveis (DCNT) no Brasil 2011-2022. Textos Básicos de Saúde, Brasilia, 2011. Série B, 148 p.

3. Pires MTB, Starling SV. Manual de urgências em pronto-socorro. ga ed. Rio de Janeiro: Guanabara Koogan; 2010. p. 655-684

4. Schoen FJ. O coração. In: Kumar V, Abbas AK, Fausto N, Aster JC. Robbins e Cotran: Bases patológicas das doenças. $8^{a}$ ed. Rio de Janeiro: Elsevier, 2010. p. 583-650.

5. Ruff CT, Braunwald E. The evolving epidemiology of acute coronary syndromes. Nat Rev Cardiol. [periódico na Internet] 2010 [citado 2012 out 20]; 8(3):140-7. Disponível em: http://www.ncbi.nlm.nih.gov/pubmed/21173793

6. Eyken EBBDV, Moraes CL. Prevalência de fatores de risco para doenças cardiovasculares entre homens de uma população urbana do Sudeste do Brasil. Cad Saude Publica. 2009; 25(1):111-123.

7. Rother ET. Revisão sistemática X revisão narrativa. [Editorial]. Acta Paul. Enferm. 2007; 20(2): v-vi.

8. Minayo MCS. 0 desafio do conhecimento: Pesquisa qualitativa em saúde. $12^{\mathrm{a}}$ ed. São Paulo: Hucitec; 2010.

9. Sampaio ES. Fatores relacionados ao retardo pré-hospitalar de pessoas com infarto agudo do miocárdio. Salvador: [s.n.], 2008. 73 p. Dissertação (Mestrado) - Universidade Federal da Bahia.

10. Damasceno CA. Fatores que influenciam o tempo de decisão de homens e mulheres com infarto do miocárdio para a procura de atendimento médico. Salvador: [s.n.], 2010. 107 p. Dissertação (Mestrado) Universidade Federal da Bahia.

11. Dessote CA. Depressão e estado de saúde percebido por pacientes com primeiro episódio de síndrome isquêmica miocárdica instável. Ribeirão Preto: [s.n.], 2010. 116 p. Tese (Doutorado) - Universidade de São Paulo.

12. Carvalho LVB. Características de pacientes após síndromes coronarianas agudas e fatores relacionados à adesão ao tratamento. São Paulo: [s.n.], 2006. 141 p. Dissertação (Mestrado) - Universidade de São Paulo.

13. Gama GGG. Fatores de Risco Cardiovascular: crenças e comportamentos de indivíduos com doença arterial coronária. Salvador: [s.n.], 2009. 91 p. Dissertação (Mestrado) - Universidade Federal da Bahia.

14. Oliveira KCS. Fatores de risco em pacientes com infarto agudo do miocárdio em um hospital privado de Ribeirão Preto-SP. Ribeirão Preto: [s.n.], 2004. 100 p. Dissertação (Mestrado) - Universidade de São Paulo.

Saúde (Santa Maria), v.38, n.2, p. 107-122, 2012. Caracterização das teses e dissertaç̧̄es de enfermagem acerca do infarto do miocárdio
15. Marinho MGR. Representação social como referência para repadronização do processo vital de clientes infartados. Florianópolis: [s.n.], 2002. 112 p. Dissertação (Mestrado) - Universidade Federal de Santa Catarina. 
16. Siviero IMPS. Saúde mental e qualidade de vida de infartados. Ribeirão Preto: [s.n.], 2003. 111 p. Tese (Doutorado) - Universidade de São Paulo.

17. Lopes JL. Banho de Aspersão e no Leito: Comparação da Percepção e do Nível de Ansiedade dos Pacientes com Infarto Agudo do Miocárdio. São Paulo: [s.n.], 2008. 188 p. Dissertação (Mestrado) Universidade de São Paulo.

18. Matos TM. Diagnóstico dos motivos de reinternação de clientes com infarto agudo do miocárdio: um estudo de enfermagem sobre o manutenção dos riscos. Rio de Janeiro: [s.n.], 2005. 113 p. Dissertação (Mestrado) - Universidade Federal do Estado do Rio de Janeiro.

19. Paiva GS. Diagnósticos de enfermagem em infartados submetidos à angioplastia coronariana com Stent. Fortaleza: [s.n.], 2007. 102 p. Dissertação (Mestrado) - Universidade Federal do Ceará.

20. Mussi FC. Padecendo a perda da espontaneidade da ação: o desconforto vivenciado por homens que sofreram infarto agudo do miocárdio. São Paulo: [s.n.], 2000. 242 p. Tese (Doutorado) - Universidade de São Paulo.

21. Stipp MAC. A saúde da mulher e os fatores de risco coronarianos: práticas de bem-estar e condições de qualidade de vida - indicativos para a assistência de enfermagem ambulatorial. Rio de Janeiro: [s.n.], 2000. 153 p. Tese (Doutorado) - Universidade Federal do Rio de Janeiro.

22. Martins DL. Perfil diagnóstico de enfermagem de pacientes acometidos por infarto do miocárdio: à luz do modelo de Florence Nightingale. João Pessoa: [s.n.], 2004. 115 p. Tese (Doutorado) - Universidade Federal da Paraíba.

23. Colombo RCR. Reabilitação no infarto do miocárdio: análise dos comportamentos relacionados a fatores de risco para coronariopatia. Ribeirão Preto: [s.n.], 2000. 319 p. Tese (Doutorado) - Universidade de São Paulo.

24. Gallani MCBJ. O exercício físico e o paciente infartado : determinantes comportamentais. São Paulo: [s.n.], 2000. 212 p. Tese (Doutorado) - Universidade de São Paulo.

25. Mussi FC. Conforto: significados e necessidades na perspectiva do paciente com infarto agudo do miocárdio. São Paulo: [s.n.], 1994. 102 p. Dissertação (Mestrado) - Universidade de São Paulo.

26. Santos FLMM. Vivenciando o infarto e redimensionando a vida. Fortaleza: [s.n.], 1998. 99 p. Dissertação (Mestrado) - Universidade Federal do Ceará.

27. Sasso GTMD. A crise como uma oportunidade de crescimento para a família que enfrenta uma doença aguda: um desafio para a enfermagem. Florianópolis: [s.n.], 1994. 228 p. Dissertação (Mestrado) Universidade Federal de Santa Catarina.

28. Siviero IMPS. 0 indivíduo infartado: a representação do impacto da sua doença. Ribeirão Preto: [s.n.], 1997. 175 p. Dissertação (Mestrado) - Universidade de São Paulo.

29. Colombo RCR. Estilo de vida e fatores de risco de pacientes com primeiro episodio de infarto agudo do miocárdio. Ribeirão Preto: [s.n.], 1995. Dissertação (Mestrado) - Universidade Federal de Ribeirão Preto.

30. Dantas RAS. Perfil de pacientes com infarto agudo do miocárdio na perspectiva do modelo de "Campo de Saúde". Ribeirão Preto: [s.n.], 1996. 158 p. Dissertação (Mestrado) - Universidade Federal de Ribeirão Preto.

31. Azevedo RCS. As possibilidades de uma abordagem cultural no cuidado ao cliente com infarto agudo do miocárdio. Florianópolis: [s.n.], 1998. 136 p. Dissertação (Mestrado) - Universidade Federal de Santa Catarina.

32. Goldmeier S. A teoria do autocuidado no manejo dos fatores de risco (obesidade, hipertensão e tabagismo) em pacientes pós-infarto agudo do miocárdio. Porto Alegre: [s.n.], 2003. Dissertação (Mestrado) Fundação Universitária de Cardiologia.

Saúde (Santa Maria), v.38, n.2, p. 107-122, 2012 Garcia, R. P., et al.

33. Farias TMVP. Reinfarto, características e fatores associados, em hospital de grande porte de Porto Alegre/RS. Porto Alegre: [s.n.], 2010. Dissertação (Mestrado) - Universidade do Vale do Rio dos Sinos. 
34. Nakajima KM. Qualidade de vida na coronariopatia: avaliação das propriedades psicométricas de instrumento específico. Campinas: [s.n.], 2006. Dissertação (Mestrado)- Universidade Estadual de Campinas.

35. Nascimento ERP. Assistência de enfermagem a indivíduos com infarto agudo do miocárdio fundamentada na teoria de Imogene King. Florianópolis: [s.n], 1991. 223 p. Tese (Doutorado) - Universidade Federal de Santa Catarina.

36. Brasil. Ministério da Educação. Coordenadoria de Aperfeiçoamento de Pessoal do Ensino Superior CAPES. Diretoria de Avaliação - DAV. Documento de área: Capes - período de avaliação 2009 - área de avaliação - Enfermagem. Brasília: Ministério da Saúde; 2009. [Relatório de avaliação trienal - 2009].

37. Custódio IL, Moreira TMM, Lima FET, Freitas MC, Lima MMR, Silva AL. Saúde do trabalhador: Caracterização das dissertações e teses nacionais de enfermagem, 2003-2007. Rev. Enferm. UERJ.[periódico na Internet] 2010 Out-Dez [citado 2012 Jul 13]; 18(4): 604-9. Disponível em: http://www.facenf.uerj.br/v18n4/v18n4a17.pdf

38. Almeida MCP, Rodrigues AP, Furegato ARF, Scochi CGS. A pós-graduação na escola de enfermagem de Ribeirão Preto-USP: Evolução histórica e sua contribuição para o desenvolvimento da enfermagem. Rev. latino-am. enfermagem [periódico na Internet]. 2002 Maio-Jun [citado 2012 Jul 13]; 10(3): 276-87. Disponível em: http://www.scielo.br/pdf/rlae/v10n3/13338.pdf

39. Mattos MA, Toledo DG, Mattos CE, Tura BR, Gadelha DNB, Filho AGS. Tendência temporal de letalidade hospitalar por infarto agudo do miocárdio. 1994-2003. Arq. bras. cardiol. 2005; 84(5):416-21

40. Arnetz JE, Winblad U, Höglund AT, Lindahl B, Spangberg K, Wallentin L et al. Is patient involvement during hospitalization for acute myocardial infarction associated with post-discharge treatment outcome? An exploratory study. Helth Expect. [periódico na Internet] 2010 Jun [citado 2012 out 25]; 13(3):298-311. Disponível em: http://www.ncbi.nlm.nih.gov/pubmed/20579120

41. Schneider DG, Manschein AMM, Ausen MAB, Martins JJ, Albuquerque GL. Acolhimento ao paciente e família na unidade coronariana. Texto \& contexto enferm. 2008; 17(1): 81-9

42. Minayo MCS, Minayo CG. Difíceis e possíveis relações entre métodos quantitativos e qualitativos nos estudos de problemas de saúde. In: Goldenberg P, arsiglia RMG, Gomes MHA. O clássico e o novo: tendências, objetos e abordagens em ciências sociais e saúde. Rio de Janeiro: Fiocruz; 2003. p. 117-42.

43. Façanha MC. Pesquisa qualitativa em saúde: Diferenças, potencialidades, limitações e possibilidades de integração com a pesquisa quantitativa. Revista de Medicina da UFC. [periódico na Internet]. 2001; [citado 2012 jul 29]; 41(1-2) [8 telas]. Disponível em: http://www.revistademedicina.ufc.br/v41/v4113.htm

44. Wright LM, Leahey M. Enfermeiras e famílias: um guia para avaliação e intervenção na família. [trad. Silvia Spada]. $4^{a}$ ed. São Paulo: Roca; 2008. 294 p.

45. Garcia RP, Budó MLD, Oliveira SG, Beuter M, Girardon-Perlini NMO. Setores de cuidado à saúde e sua inter-relação na assistência domiciliar ao doente crônico. Esc. Anna Nery Rev. Enferm. [periódico na Internet]. 2012 Abr-Jun [citado 2012 jul 29]; 16(2): 270-6. Disponível em: http://www.scielo.br/pdf/ean/v16n2/09.pdf

46. Nabão FRZ, Maruyama SAT. A experiência da enfermidade e o itinerário terapêutico vivenciado por uma pessoa com infarto. Rev. eletrônica enferm. [periódico na Internet]. 2009 [citado 2012 jul 29]; 11(1): 101-9. Disponivel em: http://www.fen.ufg.br/revista/v11/n1/v11n1a13.htm

47. Malta DC, Cezário AC, Moura L, Neto OLM, Junior JBS. A construção da vigilância e prevenção das doenças crônicas não transmissíveis no contexto do Sistema Único de Saúde. Epidemiol. serv. saúde. 2006; 15(1): 47-65.

48. WHO. World Health Organization. The Atlas of Heart Disease and Stroke. Genebra: WHO; 2004. Disponivel em: http://www.who.int/cardiovascular_diseases/resources/atlas/en/

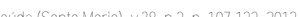
Caracterização das teses e dissertaçőes de enfermagem acerca do infarto do miocárdio

49 Alpert JS. Cardiology Patient Page: What You Need to Know If You Have Coronary Artery Disease. Cir. j. [periódico na Internet] 2010 [citado 2012 jul 29]; 124(6): 176-8. Disponível em: http://circ.ahajournals.org/content/124/6/e176.full 
50. Moser DK, Dracup K, Evangelista LS, Zambroski CH, Lennie TA, Chung ML et al. Comparison of prevalence of symptoms of depression, anxiety and hostility in elderly heart failure, myocardial infarction and coronary artery bypass graft patients. Heart lung. [periódico na Internet] 2010 Sept-Oct [citado 2012 jul 29]; 39(5): 378-85. Disponível em: http://www.ncbi.nlm.nih.gov/pmc/articles/PMC2939239/?tool=pubmed

51. Silveira CL, Budó MLD, Ressel LB, Oliveira SG, Simon BS. Apoio social como possibilidade de sobrevivência: Percepção de cuidadores familiares em uma comunidade remanescente de quilombos. Cienc. cuid. saúde. 2011; 10(3): 585-92.

52. Nascimento JS, Gomes B, Sardinha AHL. Fatores de risco modificáveis para as doenças cardiovasculares em mulheres com hipertensão arterial. Rev. RENE. 2011; 12(4): 709-15. 


\section{Raquel Pötter Garcia}

Endereço para correspondência - Rua Doutor Francisco Mariano da Rocha, 57-apto303, Bairro: Centro, CEP: 97010-170, Santa Maria, RS, Brasil.

E-mail: raquelpotter@@hotmail.com

Currículo Lattes: http://lattes.cnpq.br/7312946418733110

Recebido em 24 de agosto de 2012.

Aprovado em 12 de novembro de 2012. 